\section{Estudo da Frequiência de Cárie e Fatores Associados no Diabetes Mellitus Tipo 1}

\section{RESUMO}

Indivíduos com diabetes mellitus (DM) são mais susceptiveis a doenças da cavidade bucal, havendo controvérsias quanto à cárie. Este estudo transversal investigou a freqüência de cárie e fatores associados em amostra portadora ou não de DM tipo 1, incluindo-se 84 jovens não-diabéticos (53F / 31M) e 30 diabéticos (19F / 11M) entre 17-28 anos. O diagnóstico de cárie baseou-se no índice de CPO-D (dentes $\mathrm{C}=$ cariados; $\mathrm{P}=$ perdidos; $\mathrm{O}=$ obturados); além deste, foi obtido registro de controle de placa (RCP). Nos grupos estudados houve predomínio do sexo feminino, embora estas proporções não tenham diferido entre os grupos diabético e não-diabético. As médias de idade foram de 21,0 $\pm 2,2$ e 19,5 $\pm 1,8$ anos, respectivamente para indivíduos sem e com DM $(\mathrm{p}<0,05)$. A escolaridade foi mais elevada no grupo não-diabético, assim como o índice de CPO-D (10,5 $\pm 5,8$ vs. 6,7 $\pm 5,7 ; p<0,01)$. A análise de regressão simples $(n=114)$ mostrou associações significantes da CPO-D com idade, consumo de sacarose, número de escovações diárias, uso do fio dental, RCP e de visitas ao dentista. Em modelo de ANOVA, tendo a idade como co-variável, associaram-se significantemente à CPO-D a condição não-diabética, índice de sacarose e RCP $\left(r^{2}=0,820\right)$. No modelo que incluiu apenas o grupo com DM, tendo o índice de sacarose como co-variável, o tempo de DM, anormalidade da fundoscopia e RCP associaram-se significantemente à cárie $\left(r^{2}=0,816\right)$. A amostra de indivíduos diabéticos sugere que estes apresentam menos cáries que os não-diabéticos, apesar de realizarem maior número de refeições e menor uso de escovação e fio dental. Especula-se que a duração do DM possa contribuir para ocorrência de cárie e o consumo restrito de sacarose para menor freqüência de cárie em portadores de DM. (Arq Bras Endocrinol Metab 2006;50/3:515-522)

Descritores: Diabetes mellitus tipo 1; Cárie; Controle glicêmico; Dieta; Sacarose

\begin{abstract}
Study on the Frequency of Caries and Associated Factors in Type 1 Diabetes Mellitus.

Subjects with diabetes mellitus (DM) are more prone to certain disturbances of oral cavity but there are controversies concerning caries. This cross-sectional study investigated the frequency of caries and associated factors, in a sample of population with or without type 1 DM, including non-diabetic (53 women, 31 men) and 30 diabetic subjects (19 women, 11 men) aged 17-28 years. Diagnosis of dental caries was based on the DMF-T index ( $D=$ decay; $M=$ miss; $F=$ fill; $T=$ teeth); in addition, a plaque control record (PCR) was obtained. A preponderance of female sex was found within the groups studied but such proportions did not differ when comparing diabetic and non-diabetic groups. Mean ages were $21.0 \pm 2.2$ and $19.5 \pm 1.8$ years, respectively for subjects without and with DM $(\mathrm{p}<0.05)$. Education level was higher in the non-diabetic group as well as the DMF-T index $(10.5 \pm 5.8$ vs. $6.7 \pm 5.7$, $p<0.01)$. Linear regression analysis $(n=114)$ showed significant associa-
\end{abstract}

artigo original

Fábio M. Franco do Amaral Patricia G. de A. Ramos Sandra Roberta G. Ferreira

Programa de Pós-Graduação em Epidemiologia, Departamento de Medicina Preventiva,

Universidade Federal de São Paulo (FMFA); Estatística Aplicada - USP (PGAR); e Departamento de Medicina Preventiva, Universidade Federal de São Paulo (SRGF), São Paulo, SP.
Recebido em 09/09/05

Revisado em 16/01/06 Aceito em 12/02/06 
tions of DMF-T with age, sucrose intake, daily frequency of tooth brushing, of dental floss use, PCR and of visits to the dentist. By ANOVA model with age as a covariate the non-diabetic condition $(\mathrm{p}=$ $0.047)$, sucrose index and PCR $\left(r^{2}=0.820\right)$ were independently associated with the DMF-T. In the diabetic-specific model, with only the diabetic subjects included and sucrose index as a covariate, DM duration, fundus abnormality and PCR were significantly associated with the presence of caries $\left(r^{2}=\right.$ 0.816). The sample of type 1 diabetic subjects suggest that they are less prone to caries than non-diabetics, despite having a higher frequency of meals, less tooth brushing and dental floss use. We speculate that DM duration may contribute to the occurrence of caries and restricted sucrose consumption to lower frequency of caries in diabetic subjects. (Arq Bras Endocrinol Metab 2006;50/3:515-522)

Keywords: Type 1 diabetes mellitus; Caries; Glycemic control; Diet; Sucrose

A LÉM DE DANOS CAUSADOS pela hiperglicemia crônica nos olhos, rins, nervos e sistema cardiovascular, ocorrem distúrbios na cavidade bucal de indivíduos com diabetes (DM), sendo a doença periodontal o mais freqüente, podendo levar a perdas dentárias $(1,2)$. A cárie também traz prejuízos à estética e qualidade de vida, incidindo nas primeiras décadas de vida (3). Estudos abordando a freqüência da cárie dental em populações diabéticas são escassos; na maioria das vezes, são estudos clínicos e testam possíveis associações com o controle metabólico (4-8). Não está esclarecido se a instabilidade glicêmica no DM tipo 1, possivelmente alterando a concentração de glicose salivar e a flora bacteriana, poderia predispor ao aparecimento de cárie (8-15).

Os resultados dos estudos que mostraram os benefícios do controle glicêmico sobre as complicações microvasculares e neuropáticas do DM $(16,17)$ reforçam a importância da equipe multiprofissional de saúde na busca da normalização da glicemia e redução da morbidade. A cavidade bucal de indivíduos com DM apresenta condições propícias à ocorrência de complicações que podem assumir grandes proporções. Considerando a predisposição aumentada, particularmente naqueles com controle metabólico inadequado, é fundamental a participação do dentista nessa equipe. Dentre as alterações da cavidade bucal estão a doença periodontal e a cárie que, apesar de não comprometerem a sobrevida, podem, interferir na qualidade de vida, deteriorar o controle metabólico, agravando, assim, outras complicações crônicas diabéticas.
A cárie é uma doença multifatorial e infecciosa que leva à dissolução dos tecidos mineralizados do dente pela ação de ácidos produzidos na placa bacteriana na presença de carboidratos fermentáveis. Três são os fatores essenciais a seu aparecimento: a flora cariogênica, o substrato adequado e o hospedeiro sensível. Se um desses fatores não estiver presente a doença não se desenvolverá. Pode-se diminuir ou impedir a ação da flora bucal cariogênica por meio da ação mecânica (escovação) ou química (flúor). É possível eliminar o substrato ingerindo menor quantidade deste ou eliminando-o pela higienização. Esta é uma das mais eficazes medidas de prevenção contra a cárie e doença periodontal, devendo o hábito da escovação ser precocemente implantado. Restos alimentares nos dentes contribuem para a formação da placa bacteriana e, conseqüentemente, a instalação da cárie. Em se tratando do indivíduo diabético, especialmente o portador de DM tipo 1, este hábito se torna ainda mais relevante. A meta da Organização Mundial da Saúde - OMS, estabelecida em 2000, para crianças até 12 anos de idade foi de um número de dentes cariados, perdidos e obturados - ou índice CPO-D - inferior a 3 (18).

Até recentemente, o consumo de açúcar era contra-indicado na dieta dos indivíduos diabéticos, sendo esta conduta ainda adotada por muitos profissionais que lidam com DM. Sendo a sacarose o mais cariogênico dos açúcares, seria razoável supor que indivíduos com seu uso restrito tivessem menor ocorrência de cárie. No entanto, não há evidência de que uma dieta pobre em açúcares reverta em indiscutível redução na ocorrência de cárie nestes indivíduos (6-8,19-21). Em contrapartida, alterações quantitativas e qualitativas da saliva e da flora bacteriana da boca secundárias ao DM poderiam predispor a ocorrência de cárie (8-15). Os estudos que compararam a freqüência de cáries em indivíduos diabéticos e normoglicêmicos tiveram achados discrepantes $(4-6,11,12,14,21)$, não havendo consenso se os primeiros apresentariam ou não maior susceptibilidade.

Frente à escassez de dados particularmente nacionais sobre a relação DM e cárie, justifica-se investigar sua freqüência em amostra da nossa população, bem como fatores associados. Considerando que a faixa etária de maior acometimento de cárie é a das crianças e jovens, o foco deste estudo foi no DM tipo 1. Estes resultados podem fornecer subsídios para elaborar programas de prevenção mais dirigidos, buscar um diagnóstico mais precoce de alterações bucais no DM tipo 1 e intervir de modo mais eficaz. 


\section{MATERIAL E MÉTODOS}

O estudo foi aprovado pelo comitê de ética da Universidade Federal de São Paulo - UNIFESP. Dois grupos de indivíduos, com e sem DM tipo 1, foram convidados para participar deste estudo e todos os participantes assinaram termo de consentimento pós-informação. Os critérios de inclusão no estudo foram jovens numa faixa etária entre 17 e 28 anos de idade, de ambos os sexos, portadores e não de DM tipo 1 , diagnosticados segundo critérios da American Diabetes Association (22). Edentulismo e escolaridade inferior ao $1^{\circ}$ grau completo foram critérios de exclusão. $\mathrm{O}$ tamanho da amostra para estimar a diferença entre as médias dos 2 grupos, com $95 \%$ de confiança e erro tipo II de 0,20 , foi calculado em 36 indivíduos por grupo.

Um dos grupos foi constituído por jovens universitários convocados rotineiramente para exame admissional na UNIFESP. Estes foram seqüencialmente incluídos neste grupo exceto se referissem presença de DM tipo l. A obtenção do segundo grupo foi realizada no Centro de Diabetes da mesma instituição. Também de modo seqüencial, todos os prontuários de indivíduos diabéticos tipo $l$ a serem atendidos no serviço médico eram selecionados para exame odontológico, durante um período de 6 meses. O número calculado de indivíduos no grupo não-diabético suplantou o calculado (53 mulheres e 31 homens), enquanto que para o grupo diabético atingiu-se a totalidade de 30 indivíduos (19 mulheres e 11 homens) na faixa etária prevista.

O desenho do estudo é transversal, incluindo análises de associação da cárie ao DM. Todos os participantes foram examinados por um único cirurgião-dentista, sendo os dados colhidos de maneira sistemática. Os formulários-padrão continham dados sócio-demográficos, hábitos de vida, dieta e higiene bucal, anamnese específica e exame odontológico, cujos dados eram registrados em odontograma. No que se refere à dieta, foram obtidos dados quantitativos relativos à freqüência de alimentação entre as refeições principais, com ênfase no consumo de produtos ricos em sacarose, tais como doces, biscoitos, chocolates e refrigerantes não-dietéticos. O formulário permitiu a obtenção de variável para expressar a freqüência diária da totalidade de alimentos com sacarose, denominado para fins de análise de "índice de sacarose". Para os indivíduos com DM, havia um formulário complementar contendo dados da história da doença, complicações e exames subsidiários. $\mathrm{O}$ exame clínico bucal de todos os participantes foi realizado em cadeira odontológica sob a luz de refletor (Dabi Atlante), com o auxílio de sonda exploradora e espelho bucal número 5 (Duflex).
Com base no odontograma, que retrata detalhadamente o número de dentes, cáries e restaurações, foi obtido para cada indivíduo o índice CPOD (soma de dentes cariados, perdidos e obturados), largamente utilizado em estudos desta natureza (23). Além do CPO-D, foi obtido um índice de placa bacteriana, o registro de controle de placa ou RCP de O'Leary (24), empregando, como solução evidenciadora, uma pastilha evidenciadora à base de eritrosina $\left(\right.$ Replak $^{\circledR}$, Herpo Produtos Dentários Ltda, Rio de Janeiro). Os indivíduos foram instruídos a mastigar a pastilha até dissolver totalmente na saliva, espalhando em todas as superfícies do dente. Após enxágüe da boca evidenciaram-se as áreas dos dentes impregnadas por placa bacteriana. Após exame das 4 faces (vestibular, lingual, mesial e distal) de todos os dentes e aplica-se a fórmula:

$\mathrm{N}^{\circ}$ de superfícies coradas $/ \mathrm{N}^{\circ}$ de superfícies examinadas $\times 100=\%$ de RCP $\mathrm{N}^{\circ}$ de dentes

Após a realização dos exames, os indivíduos foram informados sobre o estado de saúde bucal e orientados quanto à higiene oral.

$\mathrm{Na}$ análise descritiva foram ressaltadas as características dos grupos diabético e não-diabéticos, comparados pelo teste $t$ de Student para médias independentes, no caso das variáveis quantitativas. As distribuições de freqüência foram analisadas pelo teste de Fisher, no caso das variáveis qualitativas. As relações entre as variáveis contidas no formulário (variáveis independentes) com o CPO-D foram analisadas pelo teste de comparação de médias independentes e, em se tratando de variáveis quantitativas, pela análise de regressão simples. Desse modo, foram selecionadas as variáveis de maior associação com o CPO-D. O efeito no CPO-D das variáveis independentes, conjuntamente, foi avaliado pelo modelo de análise de variância (ANOVA), tanto na totalidade dos indivíduos estudados, neste caso considerando a idade como co-variável, quanto no grupo dos indivíduos diabéticos, neste caso considerando o consumo de sacarose como co-variável. Neste grupo, a variação da idade foi mínima, não havendo necessidade de ser considerada co-variável. Em todas as comparações, considerou-se como significantes as probabilidades associadas aos testes inferiores a 0,05.

\section{RESULTADOS}

Em ambos os grupos de indivíduos estudados houve predomínio do sexo feminino sobre o masculino, embora estas proporções não tivessem diferido entre os 
grupos diabético e não-diabético (tabela 1 ). As médias de idade foram de $21,0 \pm 2,2$ e 19,5 $\pm 1,8$ anos, respectivamente para indivíduos sem e com DM, e esta diferença entre os grupos atingiu significância estatística $(\mathrm{p}=0,001)$. Com relação à variável cor, nota-se a inexistência de etnia amarela no grupo diabético. Conforme esperado em decorrência de peculiaridades metodológicas, os grupos diferiram quanto à escolaridade, sendo mais elevada nos não-diabéticos. Concordantemente, tanto a escolaridade do pai quanto a da mãe foram maiores neste mesmo grupo.

Na tabela 2, observa-se que indivíduos diabéticos alimentavam-se de forma mais fracionada do que os nãodiabéticos ou seja, mais freqüentemente entre as principais refeições. A freqüência de consumo diário de alimentos com sacarose se mostrou menor entre os indivíduos diabéticos quando comparados aos não-diabéticos. Nota-se, ainda, que o grupo diabético, em média, usa menos freqüentemente o fio dental que o grupo nãodiabético. Indivíduos diabéticos tenderam a escovar menos vezes os dentes do que os não-diabéticos, mas este achado não atingiu significância estatística $(\mathrm{p}=0,095)$. $\mathrm{O}$ grupo diabético informou um maior número de visitas ao dentista do que o não-diabético. Quanto ao fumo, nenhum indivíduo diabético apresentava atualmente este vício, enquanto $6 \%$ dos indivíduos não-diabéticos eram fumantes.
Na comparação do exame odontológico entre os grupos, observa-se que o índice CPO-D dos indivíduos diabéticos foi menor do que para os não-diabéticos (tabela 3). Concordantemente, o grupo diabético mais freqüentemente atingiu a meta da OMS (<3) que o não-diabético. Em relação à distribuição dos percentuais de RCP em 3 categorias, não houve diferença significante entre indivíduos diabéticos e não-diabéticos.

A análise de regressão linear simples para a totalidade dos indivíduos $(\mathrm{n}=114)$ detectou associações positivas da CPO-D com as seguintes variáveis: idade $(\mathrm{r}=0,401 ; \mathrm{p}<0,001)$, RCP $(\mathrm{r}=0,311 ; \mathrm{p}=$ $0,003)$, freqüência de consumo de alimentos ricos em sacarose $(\mathrm{r}=0,307 ; \mathrm{p}=0,001)$, de visitas ao dentista $(\mathrm{r}=0,287 ; \mathrm{p}=0,002)$, de uso do fio dental $(\mathrm{r}=0,245$; $\mathrm{p}=0,010)$ e de escovações diárias $(\mathrm{r}=0,192 ; \mathrm{p}=$ $0,040)$.

O grupo de indivíduos diabéticos estava, em média, mal controlado (hemoglobina glicada de 9,5 $\pm 2,7 \%)$. A análise de regressão simples deste grupo em particular não revelou associação do CPO-D à RCP, hemoglobina glicada e excreção urinária de albumina. No entanto, o CPO-D dos indivíduos diabéticos com retinopatia foi significantemente maior que daqueles sem esta complicação $(14,5 \pm 2,1$ vs. $5,9 \pm 5,4 ; \mathrm{p}<0,005)$.

Tabela 1. Características sócio-demográficas dos indivíduos com e sem diabetes. Dados expressos em número absoluto, percentual ou média \pm desvio-padrão.

\begin{tabular}{|c|c|c|c|}
\hline & $\begin{array}{c}\text { Não-diabéticos } \\
n=84\end{array}$ & $\begin{array}{c}\text { Diabéticos } \\
n=30\end{array}$ & Valor de $p$ \\
\hline Homens / Mulheres (\%) & $31(36,9) / 53(63,1)$ & $11(36,7) / 19(63,3)$ & 0,999 \\
\hline Idade (anos) & $21,0 \pm 2,2$ & $19,5 \pm 1,8$ & 0,001 \\
\hline $\begin{array}{l}\text { Escolaridade (\%) } \\
\text { até } 8 \text { anos } \\
9-11 \text { anos } \\
12-15 \text { anos } \\
>15 \text { anos }\end{array}$ & $\begin{array}{c}0 \\
0 \\
90,5 \\
9,5\end{array}$ & $\begin{array}{c}0 \\
83,3 \\
13,3 \\
3,3\end{array}$ & $<0,001$ \\
\hline $\begin{array}{l}\text { Cor }(\%) \\
\text { branca } \\
\text { não-branca } \\
\text { amarelo }\end{array}$ & $\begin{array}{c}75,0 \\
9,5 \\
15,5\end{array}$ & $\begin{array}{c}83,3 \\
16,7 \\
0\end{array}$ & 0,037 \\
\hline $\begin{array}{l}\text { Escolaridade do pai (\%) } \\
\text { até } 8 \text { anos } \\
9-11 \text { anos } \\
12-15 \text { anos } \\
>15 \text { anos }\end{array}$ & $\begin{array}{c}16,6 \\
26,2 \\
9,5 \\
47,6\end{array}$ & $\begin{array}{c}42,9 \\
53,5 \\
3,6 \\
0\end{array}$ & $<0,001$ \\
\hline $\begin{array}{l}\text { Escolaridade da mãe (\%) } \\
\text { até } 8 \text { anos } \\
9-11 \text { anos } \\
12-15 \text { anos } \\
>15 \text { anos }\end{array}$ & $\begin{array}{c}16,7 \\
34,5 \\
8,3 \\
40,5\end{array}$ & $\begin{array}{c}50,0 \\
46,7 \\
3,3 \\
0\end{array}$ & $<0,001$ \\
\hline
\end{tabular}


Tabela 2. Hábitos higieno-dietéticos em indivíduos com e sem diabetes. Dados expressos em percentual ou média \pm desvio padrão.

\begin{tabular}{|c|c|c|c|}
\hline & $\begin{array}{c}\text { Não-diabéticos } \\
n=84\end{array}$ & $\begin{array}{c}\text { Diabéticos } \\
n=30\end{array}$ & Valor de $p$ \\
\hline $\begin{array}{l}\text { Alimento no intervalo de } \\
\text { refeições }(\%)\end{array}$ & 61,3 & 83,3 & 0,043 \\
\hline $\begin{array}{l}\text { Número de alimentação } \\
\text { nos intervalos (vezes/dia) }\end{array}$ & $1,2 \pm 1,0$ & $1,7 \pm 0,9$ & 0,019 \\
\hline $\begin{array}{l}\text { Consumo de alimentos } \\
\text { com sacarose (vezes/dia) }\end{array}$ & $2,0 \pm 1,1$ & $0,6 \pm 0,7$ & $<0,001$ \\
\hline $\begin{array}{l}\text { Uso do fio dental (\%) } \\
\begin{array}{r}\text { Até } 1 \text { vez/dia } \\
2 \text { vezes/dia } \\
\geq 3 \text { vezes/dia }\end{array}\end{array}$ & $\begin{array}{c}69,6 \\
22,8 \\
7,6\end{array}$ & $\begin{array}{c}86,6 \\
3,3 \\
10,1\end{array}$ & 0,029 \\
\hline $\begin{array}{l}\text { Escovação (\%) } \\
\text { Até } 2 \text { vezes/dia } \\
3 \text { vezes/dia } \\
\geq 4 \text { vezes/dia }\end{array}$ & $\begin{array}{l}11,9 \\
72,6 \\
15,5\end{array}$ & $\begin{array}{l}23,3 \\
50,0 \\
26,7\end{array}$ & 0,095 \\
\hline $\begin{array}{c}\text { Visitas ao dentista (\%) } \\
\text { Até } 1 \text { vez/ano } \\
2 \text { vezes/ano } \\
\geq 3 \text { vezes/ano }\end{array}$ & $\begin{array}{c}76,2 \\
16,7 \\
7,1\end{array}$ & $\begin{array}{l}30 \\
40 \\
30\end{array}$ & $<0,001$ \\
\hline Fumo atual (\%) & 6,0 & 0 & 0,323 \\
\hline
\end{tabular}

Tabela 3. Características ao exame odontológico dos indivíduos com e sem diabetes. Dados expressos em percentual ou média \pm desvio-padrão.

\begin{tabular}{lccc}
\hline & $\begin{array}{c}\text { Não-diabéticos } \\
\mathbf{n}=\mathbf{8 4}\end{array}$ & $\begin{array}{c}\text { Diabéticos } \\
\mathbf{n}=\mathbf{3 0}\end{array}$ & Valor de $\mathbf{p}$ \\
\hline Dor de dente atual (\%) & 16,7 & 13,3 & 0,777 \\
CPO-D & $10,5 \pm 5,8$ & $6,7 \pm 5,7$ & 0,003 \\
CPO-D $<3^{*}(\%)$ & 13,1 & 36,7 & 0,013 \\
RCP & 1,7 & 3,3 & 0,490 \\
$<20 \%$ & 26,7 & 16,7 & \\
$20-40 \%$ & 71,7 & 80,0 & \\
$>40 \%$ & &
\end{tabular}

CPO-D: dentes cariados, perdidos e obturados.

* CPO-D < 3 é a meta da OMS para controle de cárie.

RCP: registro de controle de placa.

Além do DM, as variáveis selecionadas para entrar nos modelos iniciais de análise múltipla para a totalidade da amostra foram escolaridade, dor de dente, índice do consumo de sacarose, freqüêencia de uso de fio dental e de visita ao dentista e o RCP. Na tabela 4 , observa-se que a condição não-diabética, o consumo de sacarose e o RCP mostraram-se independentemente associados ao CPO-D. Tais variáveis corresponderam a um $\mathrm{r}^{2}$ de 0,82 , ou seja, explicam $82 \%$ da variabilidade total do CPO-D.

No modelo específico para indivíduos com DM (tabela 5) entraram as variáveis tempo de doença, hemoglobina glicada, retinopatia e RCP, considerando o índice de sacarose como co-variável. O tempo de $\mathrm{DM}$, presença de retinopatia e RCP mostraram-se independentemente associados ao CPO-D, com coeficiente de explicação do modelo $\left(\mathrm{r}^{2}\right)$ de 0,816 .

\section{DISCUSSÃO}

Este estudo auxiliou no esclarecimento da controversa relação entre a condição diabética e a presença de cáries em uma amostra de indivíduos brasileiros com DM tipo 1 . O grupo de indivíduos diabéticos foi comparado a uma amostra casual de jovens de faixa etária 
Tabela 4. Modelo final de ANOVA tendo a CPO-D variável resposta (contínua), considerando todos os indivíduos do estudo.

\begin{tabular}{lc}
\hline Variável & Valor de $\mathbf{p}$ \\
\hline Ausência de DM & 0,047 \\
Escolaridade & 0,074 \\
Dor de dente & 0,331 \\
Îndice de sacarose & 0,026 \\
Uso de fio dental & 0,276 \\
Número de visitas ao dentista por ano & 0,251 \\
RCP & 0,009 \\
Co-variável: Idade & 0,124 \\
R2 & 0,820 \\
\hline
\end{tabular}

As variáveis entraram no modelo como: escolaridade (9-12 anos, 13-15 anos e > 15 anos); dor de dente (não ou sim): uso de fio dental (não ou sim); índice de sacarose (não consome, 1 vez, 2 vezes, 3 ou 4 vezes e $>4$ vezes); $n^{\circ}$ de visitas ao dentista ( $<1$ vez, 1 vez, $\geq 2$ vezes); RCP $(<20 \%$, $20-40 \%$ e $>40 \%)$.

semelhante. Estes grupos diferiram quanto à distribuição da cor da pele. A inexistência da etnia amarela no grupo diabético não foi um achado inesperado, considerando que o DM tipo 1 ainda é uma doença rara entre os orientais (25). A associação da idade ao índice CPO-D na faixa etária estudada também era esperada, sendo conhecida a crescente incidência da cárie até que se atinja a idade adulta.

Uma limitação, inerente do desenho deste estudo, diz respeito à diferença na escolaridade entre os grupos. Poder-se-ia supor que nível de escolaridade mais elevado correspondesse a maior acesso à informação sobre prevenção de cáries, que poderia favorecer a menor ocorrência da doença no grupo mais diferenciado. No entanto, este fato não afetou favoravelmente os não-portadores de DM de mais alta escolaridade, uma vez que estes apresentaram CPO-D até maior que os indivíduos diabéticos. Outra limitação refere-se à confiabilidade das respostas dos indivíduos examinados; entretanto, possíveis inverdades devem ter ocorrido igualmente em ambos os grupos. Estranhamente, na análise univariada, o uso do fio dental e o número de escovações diárias mostraram-se associados à cárie. Este achado poderia tanto reforçar a hipótese da imprecisão das informações fornecidas pelos participantes como também representar uma mudança de conduta frente ao conhecimento sobre seu estado de saúde bucal comprometido. De qualquer forma, tais associações desapareceram na análise multivariada. Em geral, os dados sobre higiene bucal e visitas ao dentista indicam que os jovens de ambos os grupos devem ter recebido alguma orientação quanto aos cuidados com os dentes. O uso infreqüente do fio dental, especialmente entre os indivíduos diabéticos,
Tabela 5. Modelo final de ANOVA tendo a CPO-D variável resposta (contínua), considerando o grupo de indivíduos diabéticos.

\begin{tabular}{lc}
\hline Variável & Valor de p \\
\hline Tempo de DM & 0,008 \\
Hemoglobina glicada & 0,134 \\
Fundo de olho anormal & 0,001 \\
RCP & 0,002 \\
Co-variável: Índice de sacarose & 0,248 \\
R2 & 0,816 \\
\hline
\end{tabular}

As variáveis entraram no modelo como: tempo de DM (0-4 anos, 5-8 anos, 9-11 anos, $\geq 12$ anos), hemoglobina glicada ( $\leq 7 \%$ e $>7 \%$ ), fundo de olho anormal (não ou sim), RCP (< $20 \%, 20 \%-40 \%$ e $>40 \%$ ) e, como co-variável, índice de sacarose (não consome, 1 vez/dia, 2 vezes/dia, 3-4 vezes/dia e $>4$ vezes/dia)

DM: diabetes mellitus; RCP: Registro de Controle de Placa.

aponta a necessidade de aprimorar a educação em DM sobre os problemas odontológicos.

$\mathrm{O}$ presente estudo constatou que, de fato, jovens não-diabéticos consomem, em média, mais alimentos ricos em sacarose que os portadores da doença. Os dados referentes à associação do CPO-D com o consumo de alimentos ricos em sacarose, obtidos com a totalidade da amostra, reforçam o papel da sacarose na gênese da cárie. Porém, no modelo ajustado específico para indivíduos diabéticos, esta variável não se mostrou independentemente associada à cárie.

Outros fatores sugeridos como determinantes da maior ocorrência de cárie no grupo diabético seriam a xerostomia e concentração aumentada de glicose salivar (8-12), alterações estas observadas em indivíduos metabolicamente descompensados. Em média, os indivíduos diabéticos apresentavam hemoglobina glicada de 9,5\%, denotando controle glicêmico inadequado, mas mensurações de fluxo e concentração de glicose salivar não estavam disponíveis no presente estudo.

Apesar da menor escolaridade, inadequado controle glicêmico e do hábito de alimentar-se entre as principais refeições como exige o tratamento de sua doença, o grupo diabético apresentou menor valor médio de CPO-D. Estes achados contradizem a hipótese de que as cáries incidiriam mais comumente entre indivíduos diabéticos $(6,7,14,19)$. Pelo contrário, nosso estudo reforçaria o oposto $(5,6,20)$; uma das razões para o achado de menor número de cáries neste grupo poderia ser o uso restrito de sacarose, o mais cariogênico dos açúcares. Mesmo que o instrumento de coleta da informação não seja dos mais acurados, este foi aplicado de modo sistemático a ambos os grupos. 
A placa bacteriana desempenha papel essencial no desenvolvimento da cárie e certos agentes estão mais freqüentemente envolvidos. Estudos em jovens não-diabéticos mostraram que aqueles com níveis baixos de estreptococos mutans e lactobacilos apresentavam menor número de cáries (26-30). No entanto, nos jovens diabéticos, esta relação não é clara $(19,20,31)$, e a comparação da quantificação destas bactérias entre portadores ou não da doença também não é conclusiva (12-15). Interessantemente, a correlação entre RCP e CPO-D no presente estudo foi significante apenas entre os não-diabéticos. Isso poderia sugerir que, ainda que o jovem diabético apresentasse maior quantidade de placa bacteriana (33), esta deve possuir características menos cariogênicas, por exemplo, menor quantidade de sacarose.

No entanto, o modelo ajustado dos portadores de DM mostrou associação do CPO-D ao RCP. Este encontro não inviabiliza a hipótese da placa dos indivíduos diabéticos ser menos cariogênica, decorrente, em parte, da dieta restrita em sacarose, preconizada no seu tratamento. A ocorrência de placa (semelhante à dos não-diabéticos) é compatível com os relatos de elevada prevalência de doenças da gengiva (gengivite e doença periodontal) em pacientes diabéticos $(1,2,32)$, atribuídas ou agravadas pela presença da microangiopatia a este nível. Nossos resultados não destacam claramente o papel da placa na gênese da doença periodontal conforme a literatura o faz (33); tal discordância poderia decorrer da não-sistematização do exame gengival dos indivíduos (fora do escopo deste trabalho).

No mesmo modelo, a CPO-D não se mostrou associada à hemoglobina glicada. $\mathrm{O}$ desenho do presente estudo é inadequado para avaliar relações do tipo causa-efeito, mas, de qualquer forma, não sugeriria um papel do controle metabólico inadequado para a ocorrência de cárie. De fato, as evidências da literatura sobre a relação da hiperglicemia a distúrbios da cavidade bucal referem-se a doenças das partes moles, especialmente gengivas (34). Outro achado de interesse foi a associação da $\mathrm{CPO}-\mathrm{D}$ à retinopatia, sendo bastante escassa a literatura que explora a relação da cárie a esta complicação diabética. Considerando que este índice não reflete exclusivamente a presença da cárie, mas também dentes perdidos, é possível que seu resultado dependa - pelo menos em parte - da doença periodontal, importante causa de edentulismo. Como a microangiopatia dos tecidos gengivais pode contribuir na gênese da doença periodontal, este distúrbio da microcirculação poderia ser o elo comum da associação aqui encontrada.
Em suma, a amostra de indivíduos com DM tipo 1 estudada sugere que estes apresentem menos cáries que não-diabéticos, apesar de realizarem maior número de refeições e menor uso de escovação e fio dental. Especula-se que a duração do DM, mas não o controle glicêmico, possa contribuir para ocorrência de cárie e que o consumo restrito de sacarose possa concorrer para menor freqüência de cárie em portadores de DM.

\section{REFERÊNCIAS}

1. Bernick SM, Cohen DW, Baker L, Laster L. Dental disease in children with diabetes mellitus. J Periodontol 1975; 46:241-5.

2. Darwazeh AMG. Diabetes mellitus, dental caries and periodontal disease: evidence for a relationship. Dent Health 1990:29:3-7.

3. Messomo E. Reabilitação oral para o clínico. 2a ed. São Paulo:Editora Santos; 1994. pp. 7-60.

4. Ziskin DE, Siegel EH, Winifred C, Loughlin MD, et al. Diabetes in relation to certain oral and systemic problems. J Dent Res 1944;23:317-31.

5. Matsson L, Koch $G$. Caries frequency in children with controlled diabetes. Scand J Dent Res 1975;83(6):327-32.

6. Leeper S, Kalkwarf KL, Strom EA. Oral status of "controlled" adolescent type I diabetics. J Oral Med 1985;40(3): 127-133

7. Galea H, Aganovic I, Aganovic M. The dental caries and periodontal disease experience of patients with early onset insulin dependent diabetes. Int Dent J $1986 ; 36(4): 219-24$

8. Rosa OPS, Rocha RSS, Lopes ES. Imunoglobulinas salivares e prevalência de cárie em portadores de diabetes mellitus insulino-dependente. Rev Fac Odontol Bauru 1993; 1(1/4):18-21.

9. Marder AZ, Abelson DC, Mandel ID. Salivary alterations in diabetes mellitus. J Periodont 1975;45(9):567-9.

10. Sharon A, Ben-Aryeh H, Itzhak B, Yoram K, et al. Salivary composition in diabetic patient. $J$ Oral Med 1985;40(1):23-6.

11. Harrison R, Bowen W. Periodontal health, dental caries, and metabolic control in insulin-dependent diabetic children and adolescents. Pediat Dent 1987;9(4):283-6.

12. Twentman S, Aronsson S, Bjorkman S. Mutans streptococci and lactobacilli in saliva from children with insulindependent diabetes mellitus. Oral Microbiol Immunol $1989 ; 4(3): 165-8$.

13. Linossier A, Pizarro F, Veliz P, Arriagada M, et al. Mutans streptococci (MS) in leukemic, diabetic, and epileptic Chilean school children. J Dent Res 1981;70:403.

14. Tenovuo J, Alanen P, Larjava H, Viikari J, et al. Oral health of patients with insulin-dependent diabetes mellitus. Scand J Dent Res 1986:94:338-46. 
15. Galarza Orovio MC, Rocha RSS, Rosa OPS. Niveis salivares de estreptococos do grupo mutans e de lactobacilos em pacientes portadores de diabetes mellitus insulino-dependente. Rev Fac Odontol Bauru 1993; 1(1/4):6-12.

16. The Diabetes Control and Complication Trial Research Group. The effect of intensive treatment of diabetes on the development and progression of long-term complications in insulin-dependent diabetes mellitus. N Engl J Med 1993:329:977-86.

17. UK Prospective Diabetes Study Group. Intensive blood glucose control with sulphonylureas or insulin compared with conventional treatment and risk of complications in patients with type 2 diabetes (UKPDS 33). Lancet 1998;352:837-53.

18. Ministério da saúde. Levantamento das condições de saúde bucal da população brasileira, 2004. http://www.sobrape.org.br/falando_sobre_odontologia no brasil/dr.rodrigo b de moraes/brasil_sorridente/brasil_sorridente.html. Acessado em abril de 2005.

19. Cohen MM. Clinical studies of dental caries susceptibility in young diabetics. J Am Dent Ass 1947;34(4):239-43.

20. Sterky G, Kjellman O, Hogberg O, Lofroth AL, et al. Dietary composition and dental disease in adolescent diabetics. Acta Pediat Scand 1971;60:461-4.

21. Albrecht M, Bánóczy J, Tamás JRG. Dental and oral symptoms of diabetes mellitus. Community Dent Oral Epidemiol 1988; 16(6):378-80.

22. American Diabetes Association Expert Committee on the Diagnosis and Classification of Diabetes Mellitus. Diabetes Care 1997;20:1183-97.

23. Klein H, Palmer CE. Dental caries in American Indian children. In: Chaves MM. Odontologia social. $2 \underline{a}$ ed. Rio de Janeiro: Labor do Brasil SA; 1977. pp. 34-5.

24. O'leary TJ, Drake RB, Naylor JE. The plaque control record. J Periodont 1972;43(1):38.

25. Parky Y, Eisenbarth GS. Genetic susceptibility factors of Type 1 diabetes in Asians. Diabetes Metab Res Rev 2001;17(1):2-11.
26. Kingman A, Little W, Gomez I, Heifetz SB, et al. Salivary levels of Streptococos mutans and lactobacilli and dental caries experiences in a US adolescent population. Community Dent Oral Epidem 1988; 16:98-102.

27. Kohler B, Petterson BM, Bratthal D. Streptococcus mutans in plaque and saliva and the development of caries. Scand J Dent Res 1981;89(1):19-25.

28. Loesche WJ, Straffon LH. Longitudinal investigation of the role of Streptococcus mutans in human fissure de cay. Infect Immun 1979:26(2):498-507.

29. Van Houte J, Green DB. Relationship between the concentration of bacteria in saliva and colonization of teeth in humans. Infect Immun 1974;9(4):624-30.

30. Zickert I, Emilson CG, Krasse B. Streptococcus mutans, lactobacilli and dental health in 13-14 years old Swedish children. Community Dent Oral Epidemiol 1982; 10:77-81.

31. Wolf J. Dental and periodontal conditions in diabetes mellitus. Proc Finn Dent Soc 1977;73(suppl. 6): 1-56.

32. Mealey BL, Rethman MP. Periodontal disease and diabetes mellitus - Bidirectional Relationship. Dentistry Today 2003:107-13.

33. Wehba C, Rodrigues AS, Soares FP. Diabetes e doença periodontal: uma relação bidirecional. In: Brunetti MC, editor. Periodontia médica: uma abordagem integrada. $1 \underline{a}$ ed. São Paulo: Senac; 2003. pp. 171-95.

34. Lalla E, Lamster IB, Drury S, Fu C, et al. Hiperglicemia, glicoxidação e receptores para os produtos finais da glicação avançada: Mecanismos potenciais envolvidos nas complicações diabéticas, incluindo a periodontite associada ao diabetes. Ann Periodontol 1998;3(1):13-9.

\section{Endereço para correspondência:}

Sandra R.G. Ferreira

Departamento de Medicina Preventiva

Universidade Federal de São Paulo

Rua Botucatu 740

04023-062 São Paulo, SP

Fax: (1 1 ) 5549-5159

E-mail: ferreira@medprev.epm.br 